\title{
Accident Prevention and Traffic Pattern Analysis System for Hilly Regions
}

\author{
V. Ramachandran, R.Ramalakshmi, K. Mathankumar
}

\begin{abstract}
In hilly regions, there will be a number of curves and hairpin bends. The roadway is one of the often-used modes of transport in these regions. Accident rate and death rate in hilly regions are increasing day by day. The roads in this region will definitely have bends and steep curves; hence, it is difficult to see the vehicles coming from the opposite side. The proposed system aims in reducing the risk of driving vehicle in the terrain region with hairpin bends and steep curves. The deployed controller with ultrasonic sensor senses the vehicle coming towards the bend and intimates it to the other side of the bend or curve; it gives three stages of LED alerts to the driver driving the vehicle from the opposite side of the hairpin bend or curve. It also senses the speed of the vehicle, if the vehicle speed is high, it will alert the drivers through the buzzer. These alerts will indirectly convey the drivers to slow down the speed of the vehicle. The foremost focus of the proposed system is to prevent accidents for the drivers and passengers in order to decrease the death rates in hilly regions. This system also provides a way for analyzing the number of uphill and downhill vehicles in the hill stations by storing the data in the cloud. The analyzed data is be viewed over the internet through a web application. The web application serves as a traffic pattern analyzer for people who wish to travel by that road.
\end{abstract}

Keywords: Accident prevention, Downhill, Hilly regions, Internet of Things- IoT, Traffic pattern, Uphill.

\section{INTRODUCTION}

As per the statistics, researchers envision that by 2020 the world's motor vehicles fleet will surpass 2 million in numbers. India's automobile fleets grows at an annual rate of around 7 to $8 \%$. Road accidents contributes for over 1.2 Million mortalities worldwide, demand for the safe vehicular country is very high [1]. The privation of medical facilities during emergency and the increased number of accidents is a major concern to consider in the modern day world [2]. The accident in the hilly region occurs mainly due to the design of curved roads and hairpin bends, the lack of tracking or monitoring facility makes the accident situation worse in terrain regions [3]. The convergence of modern technologies like Internet of Things (IoT), Big data and Cloud Computing has led to solutions for traffic monitoring and analysis in smart city environments [4]. Road accidents in hilly region is always a concern due the lack of advanced infrastructure and

Revised Manuscript Received on December 16, 2019.

* Correspondence Author

V.Ramachandran*, Computer Science and Engineering, Kalasalingam Academy of Research and Education, Krishnankoil, India. Email: ramachandran.veer@gmail.com

R.Ramalakshmi, Computer Science and Engineering, Kalasalingam Academy of Research and Education, Krishnankoil, India. Email: rama@klu.ac.in

K.Mathankumar, Computer Science and Engineering, Sree Sowdambika College of engineering, Aruppukottai, India. Email: mk.mathankumar@gmail.com communication systems. Control of vehicle over the hairpin bends and steep curves in the hilly areas is a complex task without knowing the presence of a vehicle approaching in the opposite direction [5]. Many researchers have proposed Road accident detection systems and it is deployed for accident detection system in the individual vehicle [6], instead a prevention system can be proposed for the infrastructure that detects or prevents the accident in the zone. Our proposed system considers the hilly regions and aims in preventing the accidents; it also collects the traffic data and data on over speeding vehicles. The functional block diagram of the proposed system is shown in Fig 1.

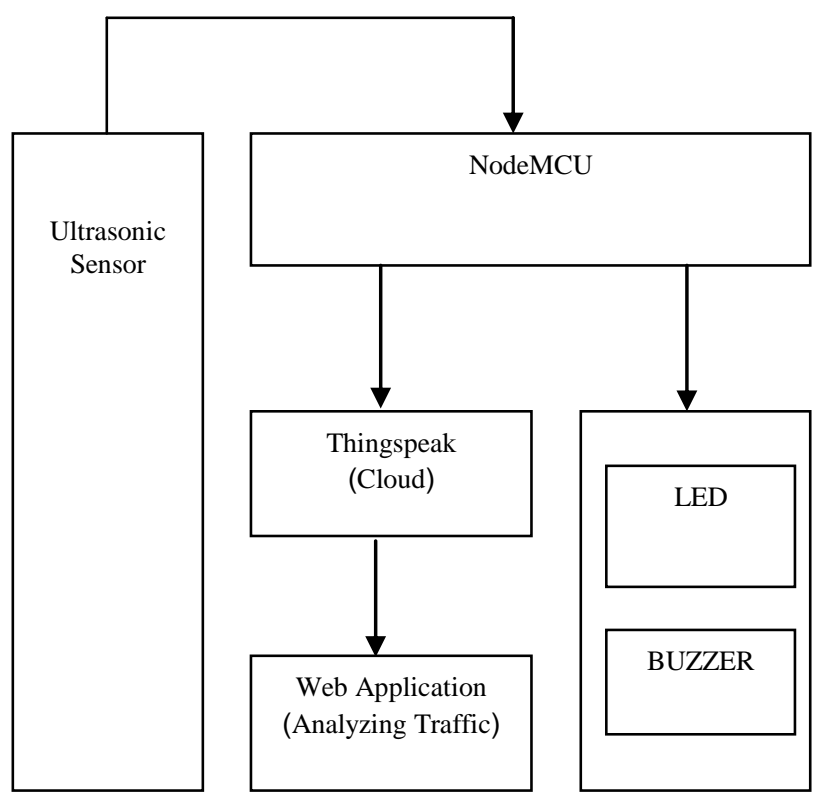

Fig. 1. Functional Block Diagram

\section{EXISTING SYSTEMS}

Driving is one of the very difficult tasks in hills. Drivers have to be alert all the time while driving in these regions. Researchers for hilly regions have proposed several accident prevention systems. Among the causes for accidents in hilly regions, the driver does not know the vehicle coming from the opposite side in curves and hairpin bends is the major cause. [11]. To avoid these problems in curves or hairpin bends a piezoelectric sensor based system is implemented [12] the system was able to alert the vehicles in the opposite side, but not able to detect vehicle when the vehicle is not over the piezo sensor. Identifying

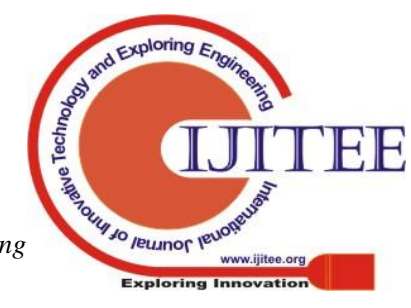


the direction of the vehicle is also a concern, as the system was not able to detect whether the vehicle is approaching the uphill or downhill direction. An automatic road accident detection and prevention system was proposed by Khalil et al., [9], the system is deployed in the individual car and only the simulation is tested. Driver fatigue and drowsy detection system is been designed and the system was able to detect only the driver drowsiness and it will not intimate about the approaching vehicle from a blind spot or curve [8]. Image processing based traffic control and accident detection system is designed [13] the processing in real time is slow and incurs high deployment cost. An instinctive speed and accident control system [14] is designed using ultrasound sensor and the system is for an individual vehicle and serves as a part of the autonomous vehicle model. Mobile application based automatic accident prevention and detection system was designed [15][16], the detection possibility of small accidents and support of the application from different mobile processors were major concerns to be addressed. A suitable cloud service is required to store the real-time data and Thingspeak an easily deployable open source service available with inbuilt lab view and MATLAB functions [18] is a right choice for swift deployment. Other than the existing research the traditional accident alert or avoidance, system includes the following.

\section{A. Convex mirror:}

Nowadays this setup is widely used in all the hilly regions. These mirrors had been installed in curves and hairpin bends where the increasing possibility of accidents. These mirrors reflect the vehicles coming in the opposite side. The main disadvantage of this system is it is difficult to keep the mirrors clean at all time. It may reduce the reflection rate and provide poor judgment to the drivers.

\section{B. Signboards.}

The signboards for hairpin bends and curves are installed in the hilly region. These sign boards for hairpin bends will just represent there are sharp bends greater than 90 degrees is coming upon the road ahead and alert to go slow. Nevertheless, this will not provide any details about the vehicles coming in the opposite side. This may provide confusion to the drivers.

\section{Horn:}

Vehicle horn is one of the most common methods used to alert the drivers in turnings like curves and hairpin bends. The drivers on both sides judge the distance of another vehicle based on the intensities of sound from their respective horns. This method is inefficient because horn sound may not be clear in raining time and causing a lot of confusion between drivers.

\section{Headlights:}

Flashing the headlights while driving is similar to the vehicle horn but it is used only in night times. This is also inefficient method because we cannot use headlights in the daytime.

\section{E. CCTV camera and LCD display:}

In this system, a CCTV camera in one side captures the vehicle and display it to the opposite side through the LCD display. The main issue in this system is it must be installed incorrect angle and if anything that falls on the lens of the camera, it will not provide a clear vision to the drivers. This may cause confusion to the drivers.

To overcome the issues in the existing methods the system using ultrasonic sensor is introduced. The sensor will sense the vehicle and calculate the distance of the vehicle, then alerts the drivers while driving in hills through LED and buzzer. This system also counts the number of uphill and downhill vehicles, used for traffic pattern analysis in hills. This system provides the way for the people to analyze the traffic through our Web Application.

\section{PROPOSED SYSTEM}

In this system, a distance of the vehicle is sensed and give three stages of alerts to the opposite side driver, these alerts are based on the distance from the vehicle to the sensor position. Using this system, the drivers can easily judge the arrival of vehicles from the other side more confidently. This system also senses the speed of the vehicle and if the vehicle speed is high, it alerts the drivers to go slow in curves and hairpin bends. In this system, the data for the number of uphill and downhill vehicles are allowed to store in the cloud and use these data for analysis purpose. This is done using Thingspeak, which is the open IoT platform with MATLAB analytics. The traffic analysis is done with the help of web application.

\section{SYSTEM DESIGN}

The design of the proposed system is categorized in as:

- Software Design

- Hardware Design

Arduino IDE 1.9.0 beta version, Thingspeak, and Windows 8.1 operating system are used in software design. In the hardware design the ultrasonic sensor, microcontroller (NodeMCU), LED's, Access points-Wi-Fi and buzzer are used.

\section{A. SOFTWARE DESIGN}

This system uses the Arduino IDE 1.9.0 beta version, Thingspeak, and Windows 8.1 operating system. The Arduino Integrated Development Environment (IDE) comprises of a text editor for writing code or instruction on to the controller. The Arduino is an Advanced Reduced instruction set computing Machine (ARM), the Arduino IDE also has a message area, a text console, a toolbar with buttons for common functions and a series of menus. It connects the microcontrollers based on Arduino hardware to upload programs and communicate with them via the USB interface.Thingspeak is a ready to use web service that provides you with API's to collect and store sensor data in the cloud for IoT applications. Thingspeak is an open source cloud platform service for IoT analytics that permits you to aggregate, visualize and also analyze live data streams in the cloud. Thingspeak is built with visualization tools that provides instant visualizations of data as soon as it is posted by the IoT devices. The ability to execute MATLAB code with in the framework makes Thingspeak a desirable platform for researchers to

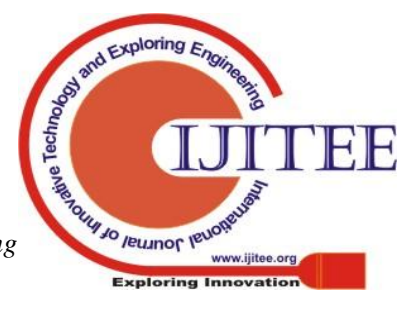


perform analysis and processing of data online as it comes in. Thingspeak is highly preferred for prototyping and proof of concept IoT systems that requires analytics.

\section{B. HARDWARE DESIGN}

This project proposes a simple approach for the implementation of accident prevention system in curves and hairpin bends on hilly regions and also in zero visibility turns using sensors, controllers, and actuators. The following Fig2 shows the block diagram of this system.

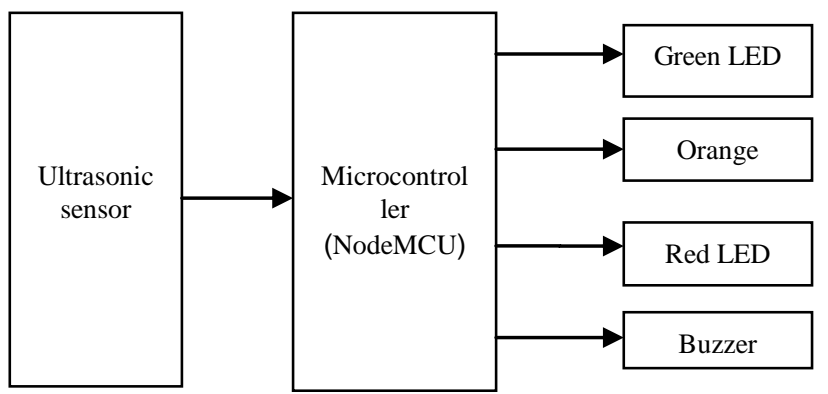

Fig. 2. Hardware Design

This system uses two ultrasonic sensors, two microcontrollers (NodeMCU board), six LED and two buzzers. Ultrasonic sensor uses the ultrasound waves to measure the distance. The sensor has a transmitter that emits ultrasonic waves and the receiver receives the wave reflected back from the obstacle or target. Using time stamp of the transmission and reception the sensor measures the distance between the sensor and target. This sensor has four pins they are Vcc(+5V DC supply), trigger, echo, and ground. It covers a maximum range of $4 \mathrm{~m}$. The sensor is connected to the microcontroller (NodeMCU) through jumpers (connecting wires). One sensor is installed on the uphill section and another sensor is installed on the downhill section. The microcontroller takes the input from the ultrasonic sensor and the output is produced through LED and buzzers. Distance is calculated by using the sensor values Speed is measured using the distance values.

\section{IMPLEMENTATION}

In this system, totally six stages(S1,S2,S3,S4,S5,S6) of alerts. Three stages $(\mathrm{S} 1, \mathrm{~S} 2, \mathrm{~S} 3)$ in one side and other three stages(S4,S5,S6) in another side.These stages are divided based on the distance from the sensor position.

a. S1 and S4 are $50 \mathrm{~cm}$ far from the sensor position.

b. $\mathrm{S} 2$ and $\mathrm{S} 5$ are $20 \mathrm{~cm}$ far from the sensor position.

c. S3 and $\mathrm{S} 6$ are $5 \mathrm{~cm}$ far from the sensor position.

The distance in the proposed system is calculated using the following formula.

$$
\begin{aligned}
\text { Distance } & =\text { Time } \\
\text { X } & =\mathrm{T}^{*} \mathrm{C}
\end{aligned}
$$

Where,

$$
\begin{aligned}
& \mathrm{X} \rightarrow \text { test distance, } \\
& \mathrm{T} \rightarrow \text { time taken, } \\
& \mathrm{C} \rightarrow \text { speed and value is } \\
& \quad \mathrm{C}=\frac{340}{2 * 10000}
\end{aligned}
$$

The velocity of sound in air is approximately $340 \mathrm{~m} / \mathrm{s}$ and Two represents the time taken by the pulse, it is actually from the ultrasonic sensor. The flowchart for calculating distance is

given in Fig 3. Distance in two positions (D1, D2) are calculated in stage S1 and S4. If the distance in the first

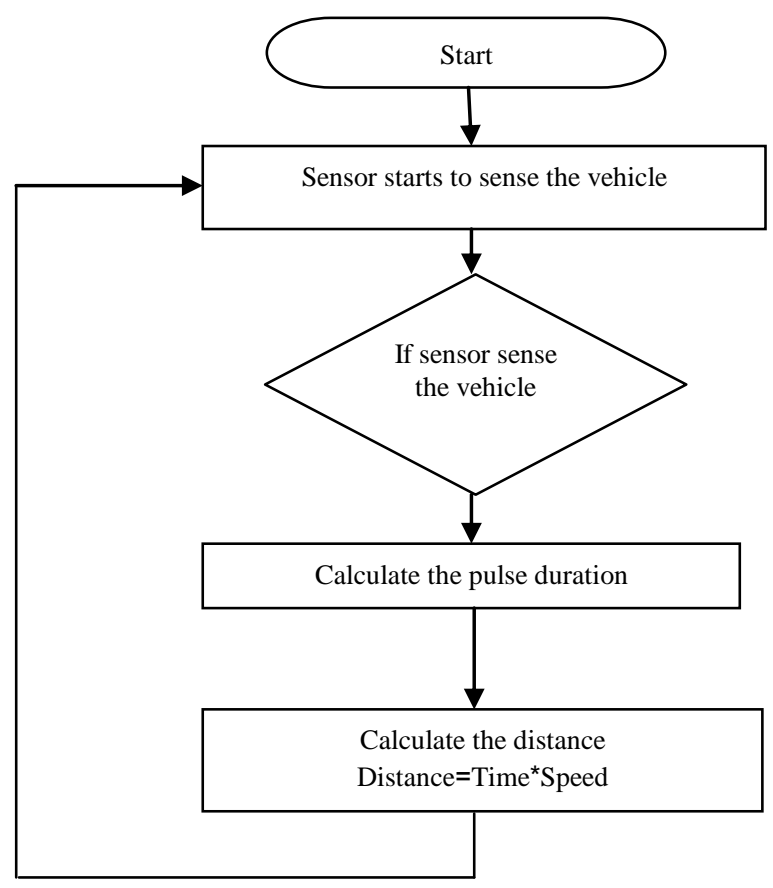

Fig. 3. Flow chart for Distance Calculation

position(D1) is greater than the distance in the second position(D2) in both the sides that is the vehicle is going outwards the following steps will be done The actual deployment of the system is shown Fig 4.

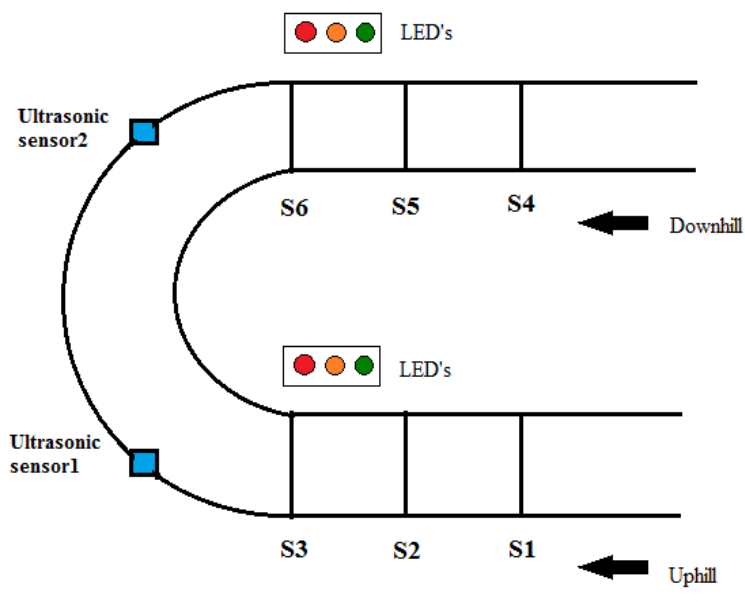

Fig. 4. Design of the proposed System

1. If the vehicle crossing the stage $S 1$, then the green light gets $\mathrm{ON}$ in the opposite side(Downhill side). If the vehicle crossing the stage $S 4$, then the green light gets $\mathrm{ON}$ in opposite side(Uphill side).

2. If the vehicle crossing the stage $\mathrm{S} 2$, then the orange light gets $\mathrm{ON}$ in opposite side(Downhill side). If the vehicle crossing the stage $\mathrm{S} 5$, then the orange light gets $\mathrm{ON}$ in opposite side(Uphill side). 


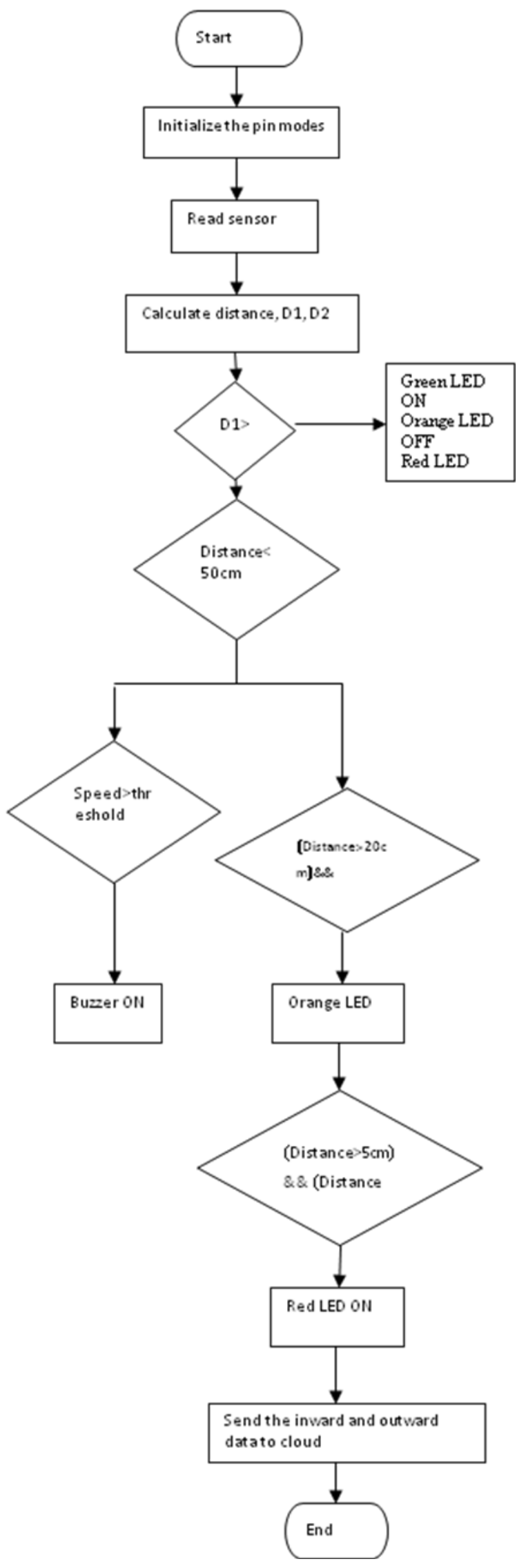

Fig. 5. Flow chart for working of this complete system

3. If the vehicle crossing the stage $\mathrm{S} 3$, then the red light gets $\mathrm{ON}$ in opposite side(Downhill side). If the vehicle crossing the stage S6, then the red light gets ON in opposite side(Uphill side).
If the distance in the first position(D1) is lesser than the distance in the second position(D2) in both the sides all the three LED's gets OFF because the vehicle is going outwards. The speed calculation in this system is done using the following formula,

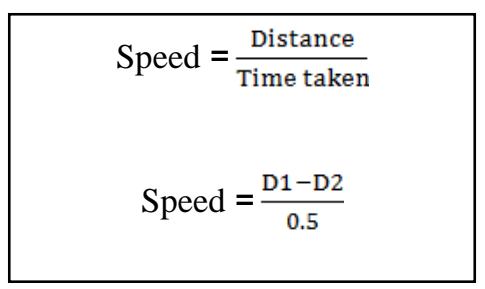

Where,

D1 is the distance in the first position

D2 is the distance in the second position

The proposed system calculates the speed in both the sides using D1 and D2 if the speed is greater than the threshold value it will alert the driver through the buzzer sound to go slowly.

The number of the uphill and downhill vehicle is stored in the cloud and we can use these data for further analysis.

The data stored in the Thingspeak cloud is retrieved using API and viewed in the Web Application by the end users, so they can easily analyze the traffic in hill regions, the flow diagram for the working of this system is shown in Fig 5. The web application or the website provides the information about the traffic in the region as in Fig 6.

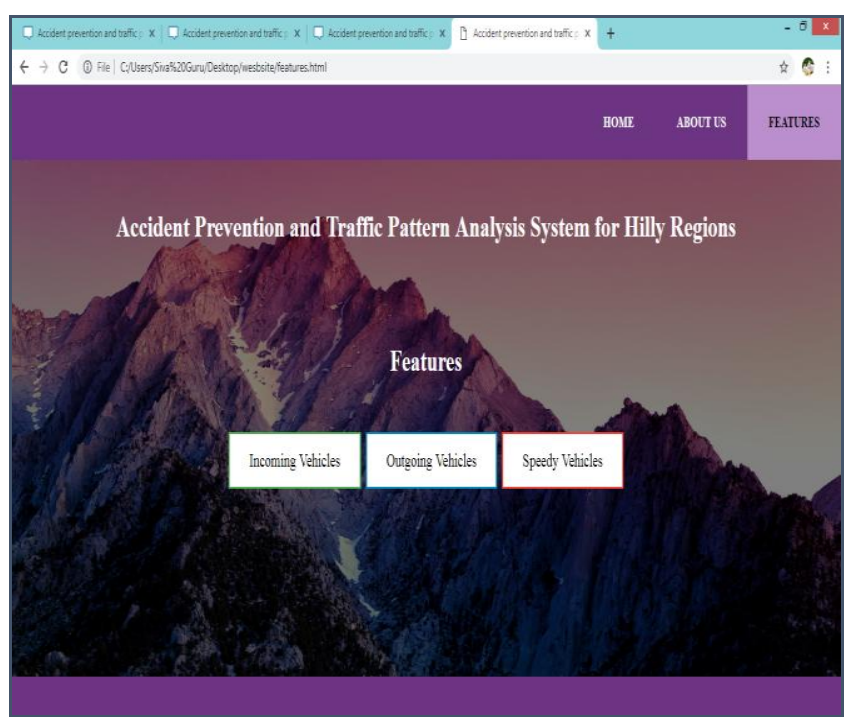

Fig. 6. Web Interface

Graphs are generated in the Thingspeak interface for uphill number of vehicles as shown in Fig 7 and downhill number of vehicles in Fig 8. 


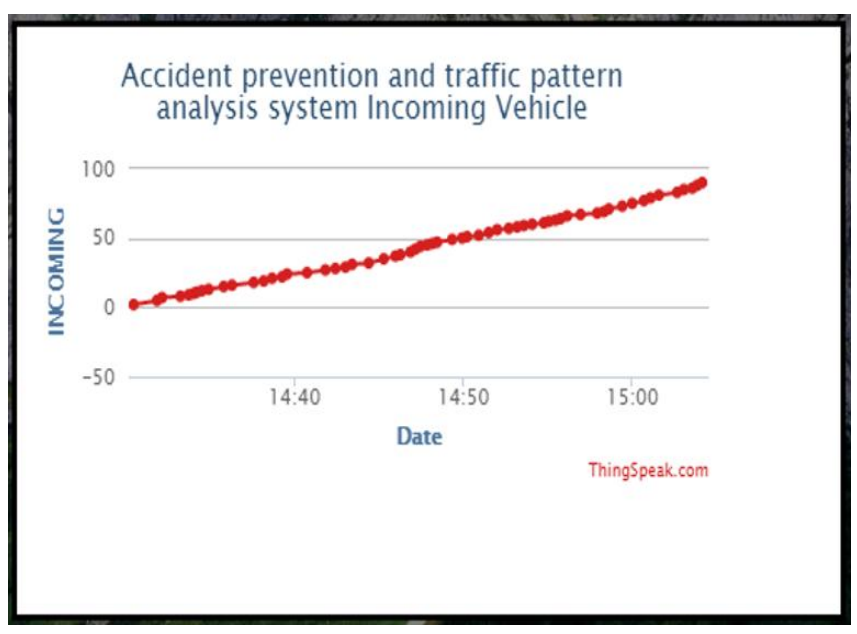

Fig. 7.Uphill vehicle pattern graph

To determine the number of speeding vehicles a graph is generated as in Fig 9 through which users will be able to predict the traffic at a given time and by analyzing the data stored the traffic pattern information high traffic time periods and low traffic time periods for uphill and downhill can also be analyzed.

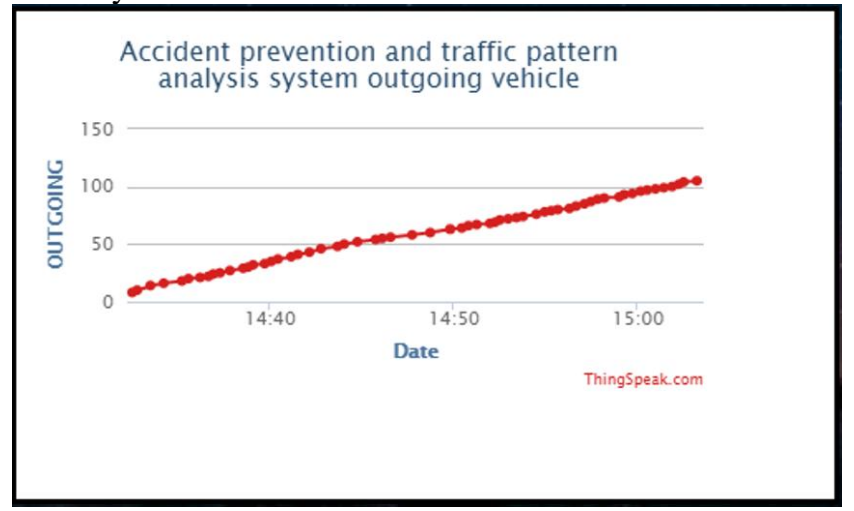

Fig. 8. Downhill vehicle patern graph

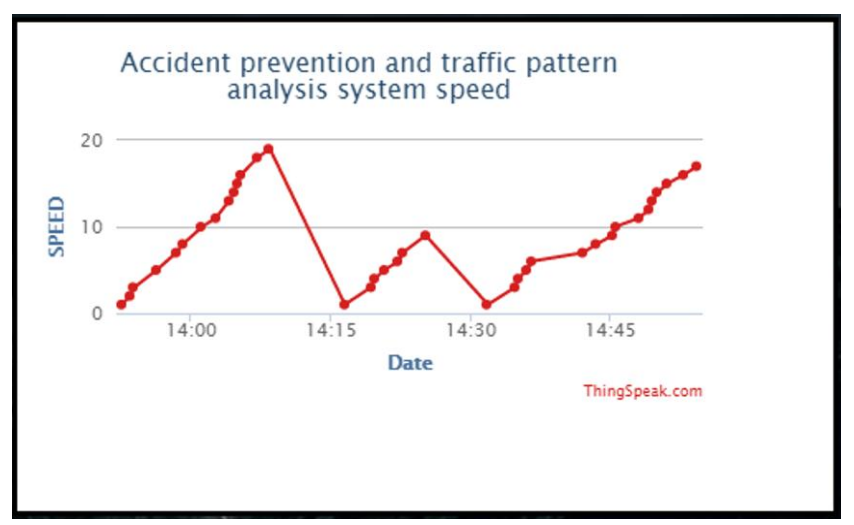

Fig. 9. Speeding vehicle pattern graph

The graphs are directly embedded to the web interface for the users using the Thingspeak API service.

\section{FUTURE DIRECTIONS}

This system requires an external power supply, implementation of a self-powered system using renewable energy like wind and solar will make the system more effective and efficient.

\section{CONCLUSION}

The ultra sound sensor based IoT system was developed and tested. The system was able to alert the vehicle approaching in the opposite direction of curve, hairpin bend or a blind spot to reduce the accident rate in hilly regions. Additionally the system was able to collect the information regarding the number of uphill and downhill vehicles in a spot and update it over the cloud along the over speeding vehicle data. This system will decrease the rate of accidents in hilly regions and helps in analyzing the traffic pattern in the region.

\section{REFERENCES}

1. Marshall, W. E. (2018). Understanding international road safety disparities: Why is Australia so much safer than the United States? Accident Analysis \& Prevention, 111, 251-265.

2. Dhanya, S., Ameenudeen, P. E., Vasudev, A., Benny, A., \& Joy, S. (2018, July). Automated Accident Alert. In 2018 International Conference on Emerging Trends and Innovations In Engineering And Technological Research (ICETIETR) (pp. 1-6). IEEE.

3. Banik, S., Agrawal, S. K., \& Singh, N. (2019). Terrain Smart Safety System with Data Hub Establishment. In Innovations in Computer Science and Engineering (pp. 479-491). Springer, Singapore.

4. Baldassarre, M. T., Caivano, D., Serrano, D., \& Stroulia, E. (2018, November). "Smart Traffic": an IoT traffic monitoring system based on open source technologies on the cloud. In Proceedings of the 1st ACM SIGSOFT International Workshop on Ensemble-Based Software Engineering (pp. 13-18). ACM.

5. Leo, J. J., Monisha, R., Sakthi, B. T. S., \& Sunder, A. J. C. (2014, February). Vehicle movement control and accident avoidance in hilly track. In 2014 International Conference on Electronics and Communication Systems (ICECS) (pp. 1-5). IEEE.

6. Khalil, U., Javid, T., \& Nasir, A. (2017, November). Automatic road accident detection techniques: A brief survey. In 2017 International Symposium on Wireless Systems and Networks (ISWSN) (pp. 1-6). IEEE.

7. Aldegheishem, A., Yasmeen, H., Maryam, H., Shah, M., Mehmood, A., Alrajeh, N., \& Song, H. (2018). Smart road traffic accidents reduction strategy based on intelligent transportation systems (tars). Sensors, 18(7), 1983.

8. Hossain, M. Y., \& George, F. P. (2018, October). IOT Based Real-Time Drowsy Driving Detection System for the Prevention of Road Accidents. In 2018 International Conference on Intelligent Informatics and Biomedical Sciences (ICIIBMS) (Vol. 3, pp. 190-195). IEEE.

9. Khalil, U., Nasir, A., Khan, S. M., Javid, T., Raza, S. A., \& Siddiqui, A. (2018, November). Automatic Road Accident Detection Using Ultrasonic Sensor. In 2018 IEEE 21st International Multi-Topic Conference (INMIC) (pp. 206-212). IEEE.

10. He, W., Yan, G., \& Da Xu, L. (2014). Developing vehicular data cloud services in the IoT environment. IEEE Transactions on Industrial Informatics, 10(2), 1587-1595.

11. Devi, B., Bavatharini, S. S., Samyuktha, G., Shobica, S., \& Sonia, E (2018, March). Voice Alert for Accident Avoidance on Merging Lanes, Blind Curves and T Junctions. In 2018 Second International Conference on Electronics, Communication and Aerospace Technology (ICECA) (pp. 418-422). IEEE

12. Balaji, P. A., Aadhivijay, R., \& Sharma, P. D. (2017, August). Hill road safety assistance using piezoelectric sensor. In 2017 International Conference on Energy, Communication, Data Analytics and Soft Computing (ICECDS) (pp. 2807-2810). IEEE.

13. Frank, A., Al Aamri, Y. S. K., \& Zayegh, A. (2019, January). IoT based Smart Traffic density Control using Image Processing. In 2019 4th MEC International Conference on Big Data and Smart City (ICBDSC) (pp. 1-4). IEEE

14. S Nagakishore Bhavanam,Vasujadevi M, "Automatic Speed Control and Accident Avoidance of Vehicle using Multi Sensors", International Conference on Innovations in Electronics and Communication Engineering (ICIECE),July 2014. 
15. White, J., Thompson, C., Turner, H., Dougherty, B., \& Schmidt, D. C. (2011). Wreckwatch: Automatic traffic accident detection and notification with smartphones. Mobile Networks and Applications, 16(3), 285-303.

16. B. Fernandes, V. Gomes, J. Ferreira, and A. Oliveira, "Mobile application for automatic accident detection and multimodal alert," in 81st Vehicular Technology Conference (VTC Spring),. IEEE, 2015, pp. $1-5$.

17. Mani, R., Solomon, N., Saranghan, M., Nagaraj, S. V., \& Ramkumar, S. (2018). ACCIDENT AVERTING SYSTEM. International Research Journal of Automotive Technology, 1(2), 9-15.

18. Ramachandran, V., Ramalakshmi, R., \& Srinivasan, S. (2018, November). An Automated Irrigation System for Smart Agriculture Using the Internet of Things. In 2018 15th International Conference on Control, Automation, Robotics and Vision (ICARCV) (pp. 210-215). IEEE.

\section{AUTHORS PROFILE}

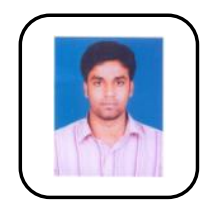

Ramachandran Veerachamy is a Full time researcher in Kalasalingam Academy of Research and Education, Lifetime member of ISTE; previously he has obtained his PG in Network Engineering in Kalasalingam University

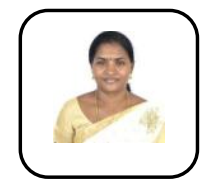

Dr Ramalakshmi Ramar is working as an Associate Professor in Kalasalingam Academy of Research and Education. She has published more than 15 Research articles in reputed conferences and high impact factor journals. Her research interest includes WSN, ADHOC networks, Data Analytics and Internet of Things.

Mathankumar $\mathrm{K}$ is working as an Assistant professor in sree sowdambika College of Engineering. Previously he has completed his M.Tech - Information technology in Kalasalingam Academy of Research and Education 